\title{
Exploration of Anti-Staphylococcus Aureus Activity and Molecular Mechanism of Wuweixiaoduyin using Network Pharmacology Anti-Staphylococcus Aureus Activity of Wuweixiaoduyin using Network Pharmacology
}

Kai Huang

Tongde Hospital Of Zhejiang Province

Bigyuan Lin

Tongde Hospital Of Zhejiang Province

Haiyong Ren

Tongde Hospital Of Zhejiang Province

Qifen Mao

Tongde Hospital Of Zhejiang Province

Qiaofeng Guo

Tongde Hospital Of Zhejiang Province

Yiyang Liu

Tongde Hospital Of Zhejiang Province

Qi You ( $\square$ qiyou0207@outlook.com )

Tongde Hospital Of Zhejiang Province

\section{Research}

Keywords: Network pharmacology, Wuweixiaoduyin, Staphylococcus aureus, Active compounds

Posted Date: June 11th, 2021

DOl: https://doi.org/10.21203/rs.3.rs-505282/v1

License: (c) (i) This work is licensed under a Creative Commons Attribution 4.0 International License.

Read Full License 


\section{Abstract}

\section{Background:}

S. aureus (Staphylococcus aureus) infection imposes a serious burden to global healthcare systems. WWXDY (Wuweixiaoduyin) is a traditional Chinese medicine, and it is usually used to treat infections in China. This study aimed to explore the active compounds, therapeutic targets, key pathways, and potential mechanisms of WWXDY in the treatment of S. aureus infection.

\section{Materials \& Methods:}

Data related to active compounds and therapeutic targets of WWXDY for treating $\mathrm{S}$. aureus were collected from DisGeNET, GeneCards, and DrugBank databases. To explore the roles of the active targets in gene function and signaling pathways, KEGG (Kyoto Gene and Genomics Encyclopedia) pathway enrichment and GO (Gene Ontology) analyses of the 122 target genes in the PPI (protein-protein interaction) network were performed. We further performed NP (network pharmacology) by using a network analyzer to screen 30 key targets.

\section{Results:}

A total 92 active compounds of WWXDY were screened. The 122 overlapped genes were found from 785 therapeutic targets and $684 \mathrm{~S}$. aureus-related genes. Besides, 92 active compounds of WWXDY, such as mandenol, ethyllinolenate, eriodyctiol, secologanic dibutylacetal_qt, etc., were identified. The PPI network of the effective ingredients of WWXDY in treating $S$. aureus infection identified the top 30 genes, including IL-6 (interleukin-6), TNF-a (tumor necrosis factor-a), VEGFA (vascular endothelial growth factor A), AKT1, CXCL8, MAPK3 (mitogen-activated protein kinase 3), TLR (toll-like receptor 4), IL-1ß, EGFR (epidermal growth factor receptor), and MMP9 (matrix metalloproteinase-9).

\section{Conclusion:}

The GO functional and KEGG pathway enrichment analyses indicated that 122 overlapped genes were mainly enriched in COVID-19, AGE-RAGE signaling pathway, C-type lectin receptor signaling pathway, Pertussis, and Chagas disease. Our findings indicated the active compounds and therapeutic targets of WWXDY in treating $\mathrm{S}$. aureus infection, as well as its potential mechanisms.

\section{Introduction}

S. aureus (Staphylococcus aureus) is a Gram-positive coccus that can cause a variety of infectious diseases, including skin and soft tissue infections, chronic osteomyelitis, and even cause toxic shock syndrome or sepsis [1-3]. Basically, $30 \%$ of healthy adults' skin and nasal cavity are colonized with S. aureus [4]. Its pathogenicity is determined by bacterial virulence, immune status, etc. Besides, the emergence of MRSA (methicillin-resistant Staphylococcus aureus) is due to increased bacterial 
resistance, resulting in the treatment failure of several common anti-staphylococcal antibiotics [5]. It may increase the risk of life-threatening infection, and impose a great burden to the society.

S. aureus can express a variety of resistance mechanisms and virulence factors, allowing it to evade host natural defenses. Particularly, S. aureus secretes numerous exotoxins, including polypeptides to damage plasma membrane [6]. The enterotoxin is a protein exotoxin, which can be released by S. aureus and mainly targets the intestines, leading to diarrhea and food poisoning. Besides, a-Hemolysin is the most characterized virulence factor of $S$. aureus. It can bind to the cell surface and form a $\beta$-barrel transmembrane pore, which can abnormally transport ions, such as $\mathrm{K}^{+}$and $\mathrm{Ca}^{2+}[7,8]$. Chronic osteomyelitis is a progressive inflammatory process, which is mainly caused by $\mathrm{S}$. aureus, and can result in bone destruction and sequestrum formation [9]. However, the management of chronic osteomyelitis has still remained a main challenge. S. aureus may cause serious damages to multi organs; thus, it is highly essential to explore its corresponding pathogenic mechanism and potential treatments.

In China, TCM (traditional Chinese medicine) has a long history in treating a variety of infectious diseases. Minami et al. demonstrated that Buzhongyiqitang, which is commonly used to improve the immunity of frail elderly people, could effectively treat nasal infection of MRSA in a mouse model [10]. Besides, extract of Angelica dahurica and Rheum officinale could also inhibit the release of inflammatory cytokines and promote wound healing, which was infected with S. aureus [11]. In TCM, WWXDY (Wuweixiaoduyin) is used to treat patients with symptoms caused by S. aureus infections, such as fever, joint pain, and swelling. Since WWXDY is composed of various medicinal materials, it is characterized by multi-pathway, -target, -component, resulting in unclear material basis and mechanisms of WWDXY in treating $\mathrm{S}$. aureus. It is highly significant to efficiently treat infectious diseases caused by $\mathrm{S}$. aureus by exploring WWDXY's active ingredients and targets.

To explore the principles of network theory and systems biology of WWDXY treatment on S. aureus, NP (network pharmacology) was applied to analyze its compound-gene/protein-pathway-disease axis, which is of great importance to describe the connections of biological systems, WWXDY, and S. aureus. In the present study, the compositions and effective components of WWXDY were screened, as well as the predicted component of target proteins. In order to cross-validate the target proteins, PPI (protein-protein interaction) network and drug-compounds-genes-disease network were cross-validated. Finally, we performed GO (Gene Ontology) and KEGG (Kyoto Encyclopedia of Genes and Genomes) enrichment pathway analyses for target proteins. This is the first study to investigate bioactive ingredients in WWXDY and reveal its potential mechanisms in treating S. aureus infection by using the NP.

\section{Materials And Methods}

\subsection{Effective components of WWXDY are screened}

All compounds of the five Chinese medicinal herbs in WWXDY were provided by the available TCMSP (Traditional Chinese Medicine Systems Pharmacology Database and Analysis Platform, 
https://tcmspw.com/index.php), and by the TCMID (Traditional Chinese Medicine Integrated Database). Based on ADME (absorption, distribution, metabolism, and excretion) parameters, bioactive components were selected. The threshold values for screening of medicinal components were considered as DL (druglikeness) $\geq 0.18$ and $\mathrm{OB}$ (oral bioavailability) $\geq 30 \%$, which contributed to compounds with higher oral absorption, utilization, and biological properties for further analysis.

\subsection{Screening of the targets of active compounds of WWXDY and S. aureus-related genes}

The SDF structure of the WWXDY components was achieved by using PubChem database. The targets with predicted scores greater than 0 were taken as the target of active compounds of WWXDY. The S. aureus-related genes were screened by DisGeNET, GeneCards, and DrugBank databases through querying the keyword of Staphylococcus aureus. Besides, Venny 2.1 software was used to filter out the co-targets between active compounds of WWXDY and S. aureus.

\subsection{Establishment of drug-compound-gene-disease network}

Based on the data of WWXDY-component-target proteins, interaction network of drug-compound-genedisease was constructed by using Cytoscape 3.8.0 software. This network includes edges and nodes, which respectively represent molecular interactions of compounds, protein targets, signaling pathways, or diseases and molecules.

Network Analyzer was used for topological analysis. The "degree" value indicated the number of correlations between the components and the targets.

\subsection{Construction of PPI network}

The retrieved active ingredient targets of WWXDY are correlated with protein targets of S. aureus by STRING database (https://string-db.org/). The species was set to "Homo sapiens". In addition, the PPI network of the effective components of WWXDY and S. aureus' protein targets was constructed based on the minimum threshold of interaction (0.4). Cystoscap 3.7.2 software was used for PPI network analysis, along with the Network Analyzer for topological analysis. Degree sequencing was performed based on the 4 parameters of degree, betweenness centrality, average path length, and so-called centrality as the reference standards. Genes with scores of top 30 were selected to draw a bar chart by R 3.6.0 programming language.

\subsection{KEGG pathway enrichment and GO analyses}

Additionally, R 3.8.1 programming language was used to carry out the KEGG pathway enrichment and GO functional analyses with a FDR (false discovery rate) $<0.2$ and P-value $<0.05$. The GO is a resource that supplies information about gene product function using ontologies to represent biological knowledge. The GO covers three domains: CC (Cellular Component), MF (Molecular Function), and BP (Biological Process). 


\section{Results}

\subsection{Effective ingredients of WWXDY and predicted targets}

WWXDY consists of Lonicera japonica, Dandelion, Semiaquilegiae Radix, Chrysanthemum, and Viola philippica. In TCMSP database, 92 compounds satisfied the criteria of $\mathrm{OB} \geq 30 \%$ and $\mathrm{DL} \geq 0.18$ and were retrieved, including 25 in Lonicera japonica, 49 in Dandelion, 7 in Semiaquilegiae Radix, 12 in Chrysanthemum, and 5 in Viola philippica (Table 1). Moreover, 785 target proteins were found among the 92 active compounds of WWXDY. The results suggested that the main active compounds of WWXDY mainly regulated inflammation-related genes.

Table 1

The active compounds and targets of WWXDY

\begin{tabular}{|lll|}
\hline Herb's Name & The number of compounds & The number of predicted targets \\
\hline Lonicera japonica & 25 & 386 \\
\hline Dandelion & 49 & 628 \\
\hline Semiaquilegiae Radix & 7 & 273 \\
\hline Chrysanthemum & 12 & 185 \\
\hline Viola philippica & 5 & 148 \\
\hline
\end{tabular}

\subsection{Retrieval Of S. Aureus-related Genes}

As shown in Table 2, a total of $683 \mathrm{~S}$. aureus-related target genes were identified after deleting repeated target genes by querying in DisGeNET, GeneCards, and DrugBank databases. The top $10 \mathrm{~S}$. aureus-related genes included NCF2, NCF1, CYBB, CYBA, CIITA, TLR2, TNF-a (tumor necrosis factor-a), MYD88, IRAK4, and ZNF341 (Table 3).

\subsection{Construction of PPI network for detecting target genes of the active compounds of WWXDY and S. aureus-related genes}

In the present study, 122 overlapped genes were selected from 785 predicted targets of WWXDY and 684 targets of S. aureus-related genes (Fig. 1). The overlapped genes included apoptosis-related genes (CASP3, CASP8, CASP1, SYK), chemokines (MMP2, MMP9), metabolic gene clusters (Nox4, GLO1), etc.

To understand the relationship between compounds of WWXDY, therapeutic targets, and S. aureus-related 
genes, a PPI network was constructed. It was composed of 459 nodes ( 5 herbs, 74 active compounds of WWXDY, 122 overlapped genes, and $1 \mathrm{~S}$. aureus-related gene) (Fig. 2). The main active compounds for the treatment of S. aureus included secologanic dibutylacetal_qt, desacetylmatricarin, chryseriol, luteolin, quercetin, acacetin, etc.

\subsection{Identification of the hub genes in the constructed PPI network}

In order to explore the interaction among 122 overlapped genes, the PPI network was constructed. We found that the network contained 122 nodes and 1,838 edges with interaction score >0.4 (Fig. 3 ). Moreover, 122 nodes represented the overlap among target proteins of active compounds of WWXDYand $\mathrm{S}$. aureus-associated genes, and 1,838 edges represented a relationship among the identified genes. Besides, IL-6 (interleukin-6), TNF-a, VEGFA (vascular endothelial growth factor A), AKT1, CXCL8, MAPK3 (mitogen-activated protein kinase 3), TLR4 (toll-like receptor 4), IL-1 $\beta$, EGFR (epidermal growth factor receptor), and MMP9 (matrix metalloproteinase-9) were the top 10 genes with the highest connection degree.

We further performed NP by using a network analyzer to screen 30 key targets. Figure 4 displays the 30 topological properties of WWXDY in the S. aureus-related genes by the PPI network.

\subsection{KEGG pathway enrichment and GO functional analyses of the target proteins}

To confirm the biological responses of S. aureus infection to WWXDY, we performed GO functional analysis of the 122 potential target genes based on MF, CC, and BP. The results showed a total of 1,988 entries. 1,815 entries were sorted in BP analysis, including leukocyte migration, reaction to lipopolysaccharide and reaction to molecule of bacterial origin. In CC analysis, 69 obtained cases were involved in membrane regions membrane microdomains, membrane rafts, etc. Besides, 104 entries were screened after MF analysis. These cases were related to endopeptidase activity, binding receptors to cytokines, serine-type peptidase activity, etc. The top $20 \mathrm{GO}$ terms are listed in Fig. 5.

Additionally, we performed the KEGG pathway enrichment analysis to reveal the biological processes. It was revealed that the genes mentioned above were enriched in 145 pathways with P-value $<0.05$ according to the number of the target genes. The top 5 pathways included COVID-19, AGE-RAGE signaling pathway, Pertussis, Chagas disease and CLR (C-type lectin receptor) signaling pathway (Fig. 6).

\section{Discussion}

S. aureus is one of the common pathogenic bacteria that causes numerous infectious diseases, such as mastitis, chronic osteomyelitis, pneumonia, etc. [12-14]. The results of previous research have shown 
that the treatment of $\mathrm{S}$. aureus is accompanied by several drawbacks. Although the antibiotic treatment may be significant for bacterial infections to a certain extent, the abuse of antibiotics not only leads to the emergence of drug-resistant strains-MRSA, but also causes antibiotic residues, seriously threatening food safety [15]. Thus, there is a main challenge to find out an appropriate substitute for antibiotic treatment of $\mathrm{S}$. aureus infection.

With the increased clinical application of TCM and its extracts in prevention and treatment of S. aureus infection, its therapeutic effects have been extensively confirmed. Lan et al. demonstrated that flavonoids from Artemisia rupestris L., a traditional Chinese herb, had antibacterial effects against S. aureus [16].The antibacterial activity of Sophora flavescens extract, Angelica sinensis extract, S. flavescens extract and herb pair $A$. sinensis was investigated, and it was found that $S$. flavescens extract showed a strong antibacterial effect on Chalmers, Escherichia coli, Shigella castellani, and Staphylococcus aureus [17]. In TCM, Scutellaria baicalensis is widely used to treat infections with a symptom of fever. As an active compound of Scutellaria baicalensis, baicalin can effectively reduce the virulence of $\mathrm{S}$. aureus, and cooperate with penicillin $\mathrm{G}$ in anti-infection treatment with an improved efficacy of 75\% [18]. WWXDY is a kind of TCM, which is often used to treat bacterial infection. It is composed of Lonicera japonica, Dandelion, Semiaquilegiae Radix, Chrysanthemum, and Viola philippica. In our previous study, we attempted to assess the clinical efficacy of the combination of TCM and Western medicine on the traumatic chronic tibial osteomyelitis (CO). We found that combination of the TCM and Western medicine could mitigate the local lesion of traumatic $\mathrm{CO}$ and ameliorate the general status [19]. However, the active compounds of WWXDY and its potential molecular mechanisms for the treatment of $\mathrm{S}$. aureus have still remained elusive.

In the current study, 92 active compounds of WWXDY, such as mandenol, ethyllinolenate, eriodyctiol, secologanic dibutylacetal_qt, etc. were identified. In addition, 785 target genes of WWXDY and 683 S. aureus-related genes were predicated using the mentioned databases. Importantly, 122 overlapped genes between WWXDY and S. aureus-related genes mainly belonged to apoptosis-related genes (CASP3, CASP8, CASP1, and SYK), chemokines (MMP2, MMP9), and metabolic genes (Nox4, GLO1). S. aureus infection has been proved to affect cell proliferation, apoptosis, metabolism, as well as secretion of chemokines [20-22]. Therefore, WWXDY has a great potential in the treatment of S. aureus infection.

Based on the constructed PPI network, we found that secologanic dibutylacetal_qt, desacetylmatricarin, chryseriol, luteolin, quercetin, and acacetin were the main active compounds for the treatment of $S$. aureus infection. These ingredients, e.g. chryseriol, luteolin, quercetin, and acacetin, have been proved to improve the treatment of S. aureus infection [16, 23-25].

The promising applications of PPI networks to disease datasets are mainly 4 areas: (i) identification of disease-related subnetworks, (ii) network-based disease classification, (iii) the identification of genes and proteins associated with diseases, and (iv) the study of network properties and their relation to disease status. By constructing PPI network, it was found that IL-6, TNF-a, VEGFA, AKT1, CXCL8, MAPK3, TLR4, IL$1 \beta$, MMP9 and EGFR were the top 10 hub genes, and they were mainly enriched in inflammation-related 
signaling pathways. It is significant to deepen the understanding of the functional relationship between proteins. In macrophages and epithelial cells, $\mathrm{S}$. aureus could enhance the expression levels of proinflammatory cytokines, including IL-1 $\beta$, IL-6, and TNF-a [26, 27], which were highly correlated with therapeutic targets of WWXDY. Wu et al. [28] demonstrated that Chryseriol, which is a main active compound of WWXDY, could significantly inhibit IL-6, TNF-a, and IL-1 in inflammatory diseases.

To explore the roles of the active targets in gene function and signaling pathways, KEGG pathway enrichment and GO functional analyses of the 122 target genes in the PPI network were performed. The effects of WWXDY in the treatment of $S$. aureus infection are mainly attributed to the regulation of endopeptidase activity, binding receptors to cytokines, and serine-type peptidase activity. Besides,KEGG pathway enrichment analyses indicated that identified genes were mainly enriched in COVID-19, CKR signaling pathway, AGE-RAGE signaling pathway, Pertussis, and Chagas disease. The advantages of TCM in the treatment of COVID-19 include effective relief of symptoms, improvement of cure rate, reduction of mortality, and promotion of rehabilitation [29]. COVID-19 patients have a high probability of co-infection with S. aureus [30]. Our data suggest that WWXDY may provide a new therapeutic option for the treatment of COVID-19. The AGE-RAGE signaling pathway was firstly recognized to be involved in S. aureus infection. The complications of atherosclerosis and nephropathy in type 2 diabetes (T2DM) might be treated with TCM mainly through the AGE-RAGE signaling pathway [31]. We found that WWXDY could treat $\mathrm{S}$. aureus infection, which was correlated with the regulation of AGE-RAGE signaling pathway.

\section{Conclusions}

In summary, our findings revealed the effective ingredients of WWXDY and their targets in the treatment of S. aureus infection. Secologanic dibutylacetal_qt, desacetylmatricarin, chryseriol, and other main active compounds may play therapeutic roles in regulating their targets, including IL-6, TNF, VEGFA, AKT1, CXCL8, etc. However, further studies should be performed to clarify the specific therapeutic mechanisms of the active compounds of WWXDY. The results of the current research may be significant for effective drug selection and accurate treatment of $\mathrm{S}$. aureus infection.

\section{Abbreviations}

WWXDY - Wuweixiaoduyin

KEGG - Kyoto Gene and Genomics Encyclopedia

GO - Gene Ontology

PPI - Protein-Protein Interaction

NP - Network Pharmacology

IL-6 - Interleukin-6 
TNF-a - Tumor Necrosis Factor- $a$

VEGFA - Vascular Endothelial Growth Factor A

MAPK3 - Mitogen-Activated Protein Kinase 3

TLR - Toll-Like Receptor 4

EGFR - Epidermal Growth Factor Receptor

MMP9 - Matrix Metalloproteinase-9

S. aureus - Staphylococcus aureus

MRSA - Methicillin-Resistant Staphylococcus Aureus

TCM - Traditional Chinese Medicine

TCMSP - Traditional Chinese Medicine Systems Pharmacology Database and Analysis Platform

TCMID - Traditional Chinese Medicine Integrated Database

ADME - Absorption, Distribution, Metabolism, and Excretion

DL - Drug-Likeness

OB - Oral Bioavailability

FDR - False Discovery Rate

CC - Cellular Component

MF - Molecular Function

BP - Biological Process

CLR - C-type Lectin Receptor

\section{Declarations}

\section{Funding}

This study was financially sponsored by Zhejiang Medical and Health Science and Technology Program (Grant No. 2020RC048), Natural Science Foundation of Zhejiang Province (Grant No. LY20H270002), National Natural Science Foundation of China (Grant No. 81603644), and Zhejiang TCM Science and Technology Program (Grant No. 2020ZQ006). 


\section{Ethics approval and consent to participate}

Ethical approval was obtained from IEC of Tongde Hospital of Zhejiang Province.

\section{- Authors' contributions}

ABT- Concepts, Design, Data analysis, Statistical analysis, Manuscript preparation, Manuscript review, Guarantor

ZNS - Concepts, Design, Data analysis, Statistical analysis, Manuscript preparation, Manuscript review, Guarantor

GBK - Concepts, Design, Data analysis, Statistical analysis, Manuscript preparation, Manuscript review, Guarantor

APU -- Definition of intellectual content, Literature search, Experimental studies, Data acquisition, Manuscript editing, Guarantor

AST-Definition of intellectual content, Literature search, Experimental studies, Data acquisition, Manuscript editing

ZKS- Data analysis, Statistical analysis, Manuscript editing, Guarantor

\section{- Acknowledgements}

None

\section{- Data availability statement}

Data sharing is not applicable to this article as no new data were created or analyzed in this study. All the generated data are available in this article itself. The raw data that support the findings of this study are available from the corresponding author upon reasonable request.

\section{Conflicts of Interest Statement}

The authors of this article certify that they have no affiliations with or involvement in any organization or entity with any financial interest or non-financial interest in the subject matter or materials discussed in this manuscript that could have appeared to influence the work reported in this paper.

\section{Consent to Publish}

Not Applicable

\section{References}


1. Josse J, Velard F, Gangloff SC. Staphylococcus aureus vs. Osteoblast: Relationship and Consequences in Osteomyelitis. Front Cell Infect Microbiol. 2015;5:85.

2. Olaniyi R, et al. Staphylococcus aureus-Associated Skin and Soft Tissue Infections: Anatomical Localization, Epidemiology, Therapy and Potential Prophylaxis. Curr Top Microbiol Immunol. 2017;409:199-227.

3. Kim D, et al., Toxic Shock Syndrome Toxin 1-Producing Methicillin-Resistant Staphylococcus aureus of Clonal Complex 5, the New York/Japan Epidemic Clone, Causing a High Early-Mortality Rate in Patients with Bloodstream Infections. Antimicrob Agents Chemother, 2019. 63(11).

4. Melehani JH, Duncan JA. Inflammasome Activation Can Mediate Tissue-Specific Pathogenesis or Protection in Staphylococcus aureus Infection. Curr Top Microbiol Immunol. 2016;397:257-82.

5. Hassoun A, Linden PK, Friedman B. Incidence, prevalence, and management of MRSA bacteremia across patient populations-a review of recent developments in MRSA management and treatment. Crit Care. 2017;21(1):211.

6. Ryu S, et al. Colonization and infection of the skin by S. aureus: immune system evasion and the response to cationic antimicrobial peptides. Int J Mol Sci. 2014;15(5):8753-72.

7. Putra I, et al. Staphylococcus aureus alpha-hemolysin impairs corneal epithelial wound healing and promotes intracellular bacterial invasion. Exp Eye Res. 2019;181:263-70.

8. Menestrina G, et al. Ion channels and bacterial infection: the case of beta-barrel pore-forming protein toxins of Staphylococcus aureus. FEBS Lett. 2003;552(1):54-60.

9. Lan Y, et al. NEMO-binding domain peptide ameliorates inflammatory bone destruction in a Staphylococcus aureus-induced chronic osteomyelitis model. Mol Med Rep. 2019;19(4):3291-7.

10. Minami M, Konishi T, Makino T. Effect of Hochuekkito (Buzhongyiqitang) on Nasal Cavity Colonization of Methicillin-Resistant Staphylococcus aureus in Murine Model. Medicines (Basel), 2018. 5(3).

11. Yang WT, et al. Effects of Angelica dahurica and Rheum officinale Extracts on Excisional Wound Healing in Rats. Evid Based Complement Alternat Med. 2017;2017:1583031.

12. Hoekstra J, et al. Differences between Staphylococcus aureus lineages isolated from ovine and caprine mastitis but not between isolates from clinical or subclinical mastitis. J Dairy Sci. 2019;102(6):5430-7.

13. Conlon BP, et al. Persister formation in Staphylococcus aureus is associated with ATP depletion. Nat Microbiol. 2016;1:16051.

14. Yu B, et al. Geniposide attenuates Staphylococcus aureus-induced pneumonia in mice by inhibiting NF-kB activation. Microb Pathog. 2017;112:117-21.

15. Lakhundi S, Zhang K. Methicillin-Resistant Staphylococcus aureus: Molecular Characterization, Evolution, and Epidemiology. Clin Microbiol Rev, 2018. 31(4).

16. Lan JE, et al., Flavonoids from Artemisia rupestris and their synergistic antibacterial effects on drugresistant Staphylococcus aureus. Nat Prod Res, 2019: p. 1-6. 
17. Han C, Guo J. Antibacterial and anti-inflammatory activity of traditional Chinese herb pairs, Angelica sinensis and Sophora flavescens. Inflammation. 2012;35(3):913-9.

18. Zhang $\mathrm{H}$, et al. Baicalein mediates protection against Staphylococcus aureus-induced pneumonia by inhibiting the coagulase activity of vWbp. Biochem Pharmacol. 2020;178:114024.

19. Huang $\mathrm{K}$, et al. Research on the clinical efficacy of the combination of Chinese traditional medicine and western medicine on the chronic traumatic tibial osteomyelitis. Pak J Pharm Sci. 2018;31(6(Special)):2841-5.

20. Xu J, et al. Mangiferin Inhibits Apoptosis and Autophagy Induced by Staphylococcus aureus in RAW264.7 Cells. J Inflamm Res. 2020;13:847-57.

21. Favazzo LJ, et al., The Response of nor and nos Contributes to Staphylococcus aureus Virulence and Metabolism. J Bacteriol, 2019. 201(9).

22. Saleh K, et al. Inflammation Biomarkers and Correlation to Wound Status After Full-Thickness Skin Grafting. Front Med (Lausanne). 2019;6:159.

23. Qian W, et al. Antimicrobial mechanism of luteolin against Staphylococcus aureus and Listeria monocytogenes and its antibiofilm properties. Microb Pathog. 2020;142:104056.

24. Wang $Z$, et al. Characterization and bacteriostatic effects of $\beta$-cyclodextrin/quercetin inclusion compound nanofilms prepared by electrospinning. Food Chem. 2021;338:127980.

25. Bi C, et al., Acacetin Protects Mice from Staphylococcus aureus Bloodstream Infection by Inhibiting the Activity of Sortase A. Molecules, 2016. 21(10).

26. Deramaudt TB, et al. Sulforaphane reduces intracellular survival of Staphylococcus aureus in macrophages through inhibition of JNK and p38 MAPK-induced inflammation. Int J Mol Med. 2020;45(6):1927-41.

27. Wang $\mathrm{H}$, et al. Selenium ameliorates Staphylococcus aureus-induced inflammation in bovine mammary epithelial cells by inhibiting activation of TLR2, NF-KB and MAPK signaling pathways. BMC Vet Res. 2018;14(1):197.

28. Wu JY, et al. Chrysoeriol ameliorates TPA-induced acute skin inflammation in mice and inhibits NFKB and STAT3 pathways. Phytomedicine. 2020;68:153173.

29. Ren X, et al. Identifying potential treatments of COVID-19 from Traditional Chinese Medicine (TCM) by using a data-driven approach. J Ethnopharmacol. 2020;258:112932.

30. Adeiza SS, Shuaibu AB, Shuaibu GM. Random effects meta-analysis of COVID-19/S. aureus partnership in co-infection. GMS Hyg Infect Control. 2020;15:Doc29.

31. He D, et al., A Network Pharmacology-Based Strategy For Predicting Active Ingredients And Potential Targets Of LiuWei DiHuang Pill In Treating Type 2 Diabetes Mellitus. 2019. 13: p. 3989-4005.

\section{Tables}

Tables 2 and 3 are not available with this version. 
Figures

\section{WWXDY \\ S. aureus}

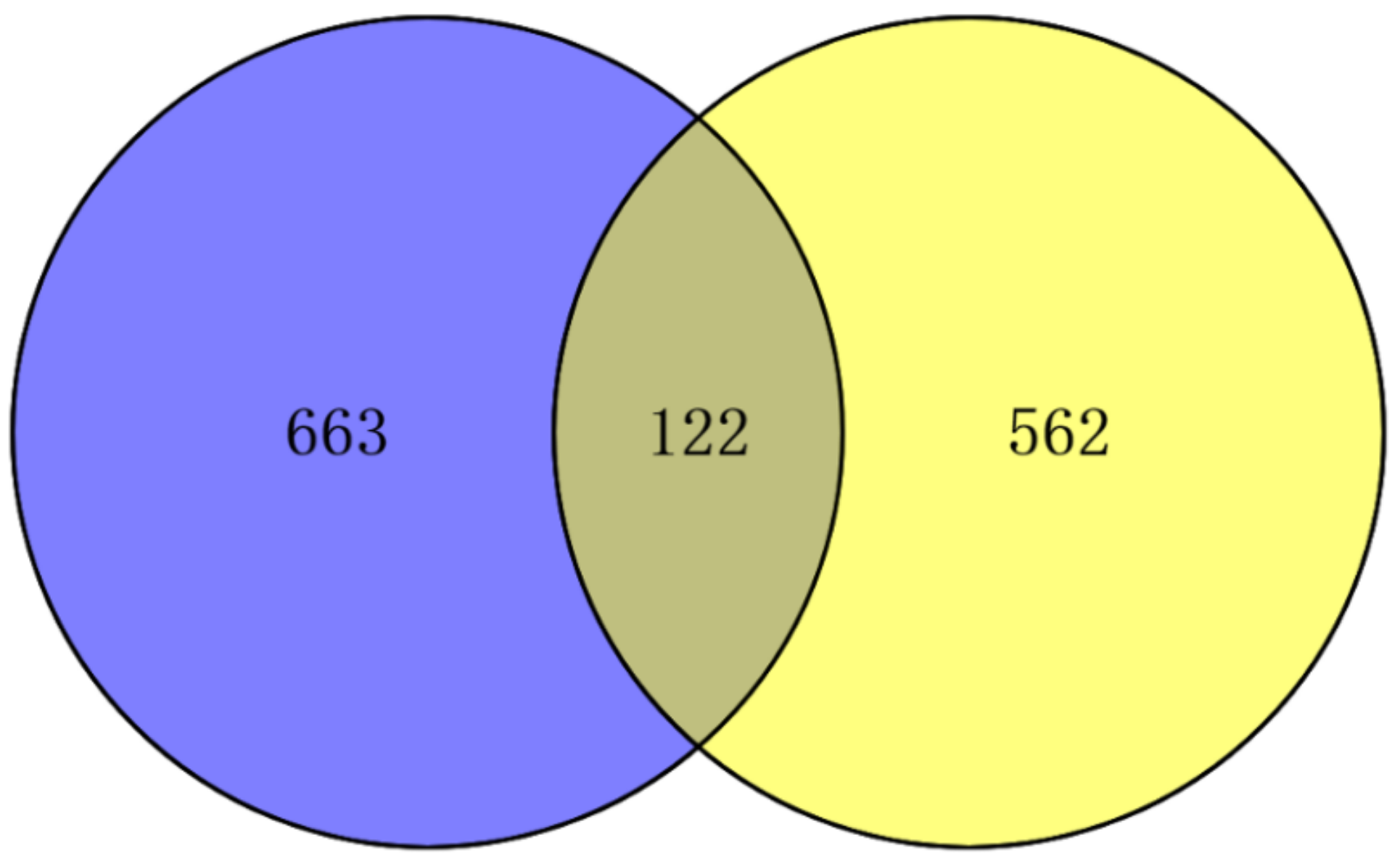

Figure 1

Venn plot of the overlapped genes between targets of the active compounds of WWXDY and S. aureusrelated genes. 


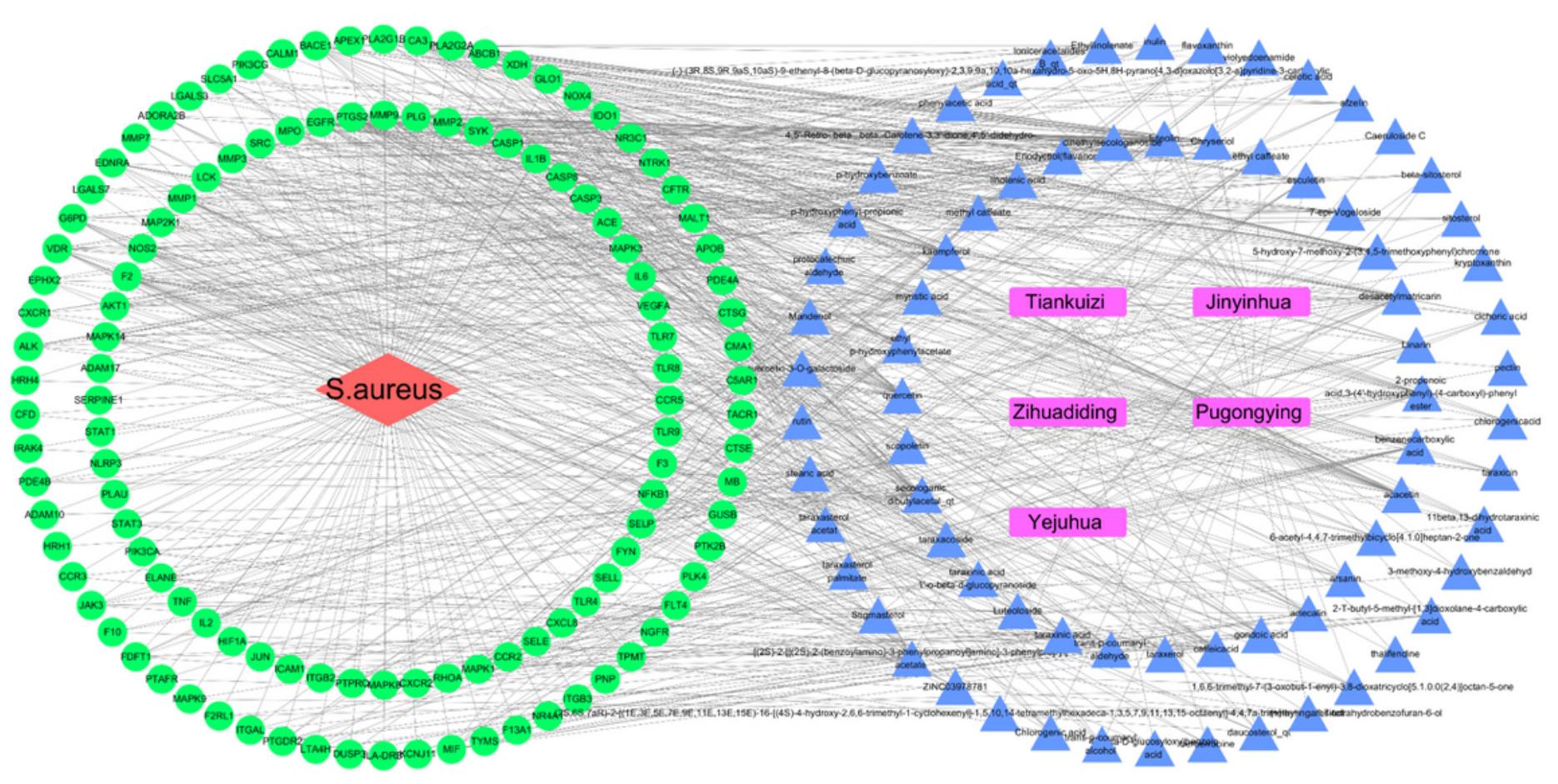

\section{Figure 2}

A PPI network illustrating the relationship between compounds of WWXDY, therapeutic targets, and S. aureus-related genes. Purple represents herbs, blue indicates 74 active ingredients in WWXDY (18 deleted active targets are not correlated with disease's target genes), green represents 122 overlapped genes, and red shows S. aureus-related genes.
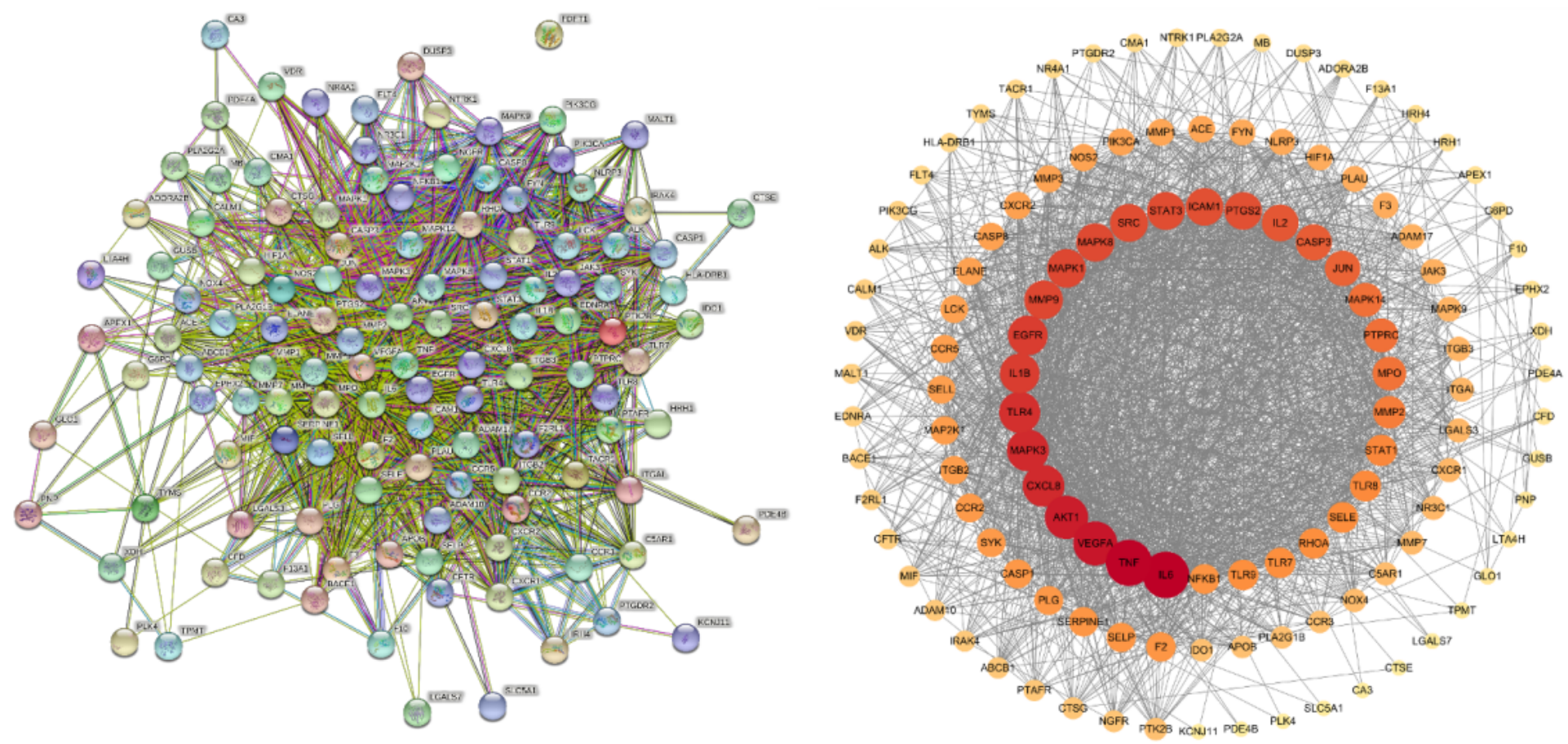

Figure 3 
Illustration of PPI network.

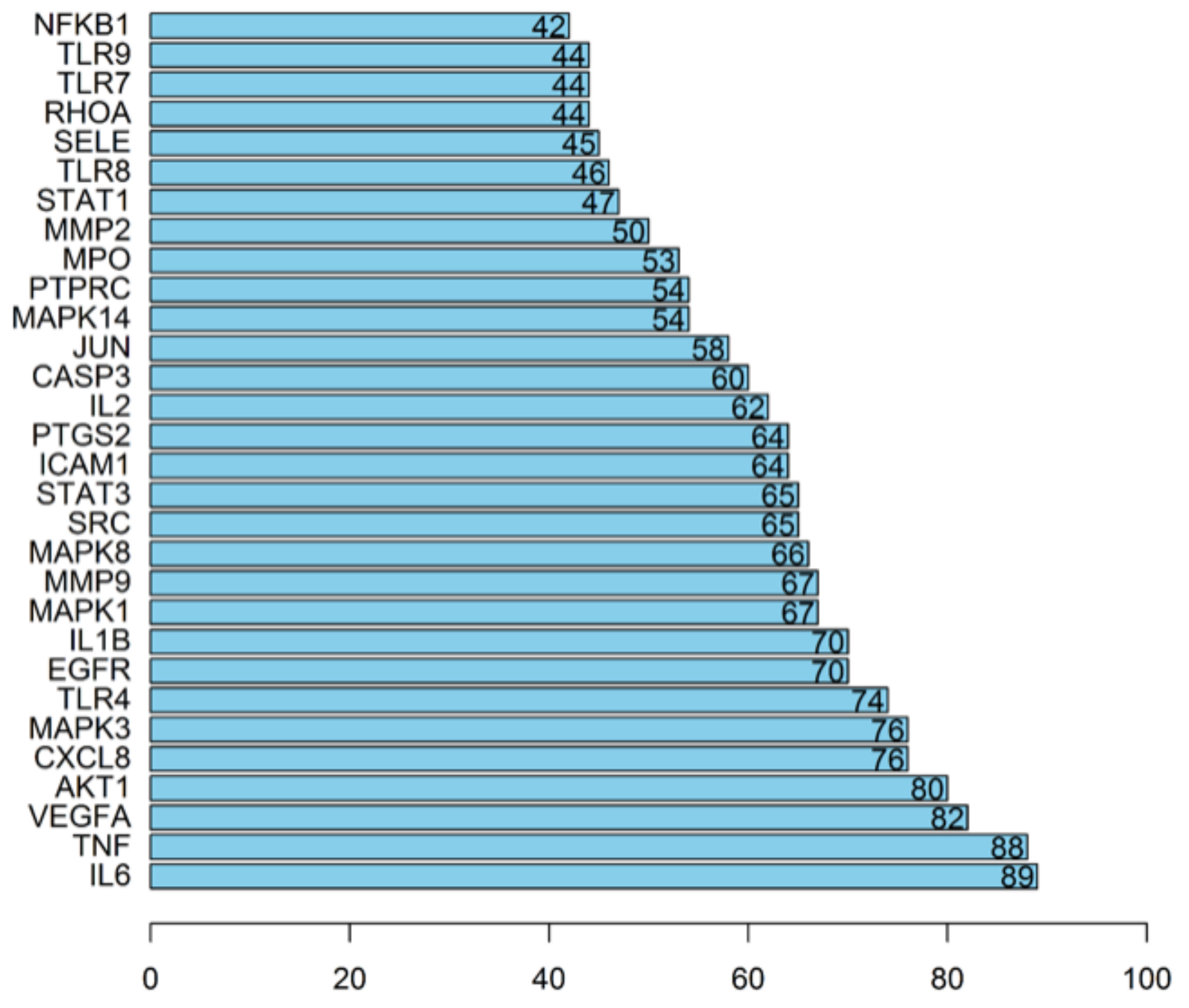

Figure 4

PPI network of the 30 overlapped genes between target genes of the active compounds of WWXDY and S. aureus-related genes. 


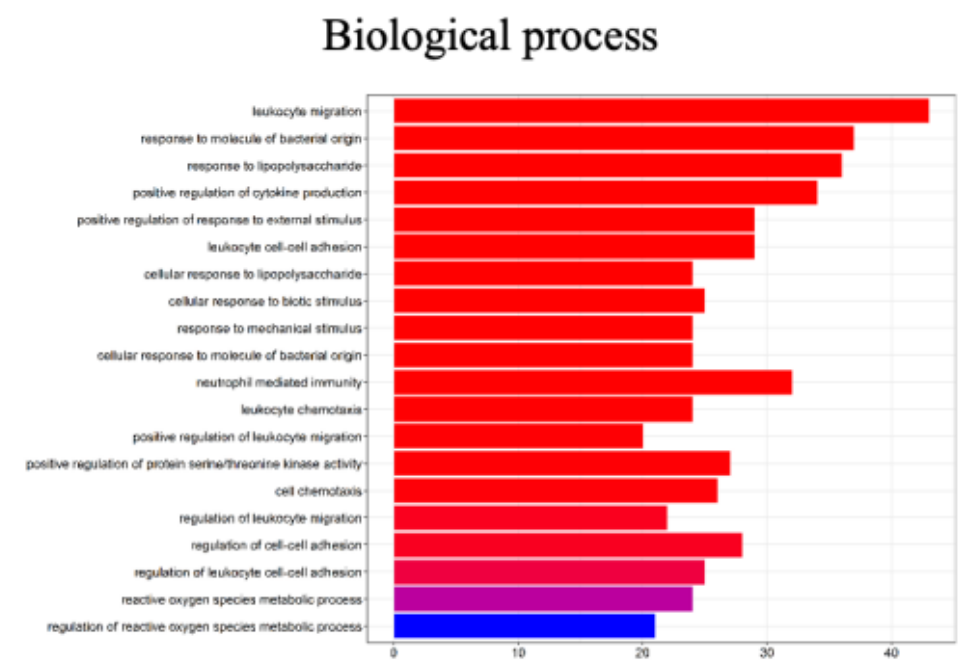

\section{Cell components}

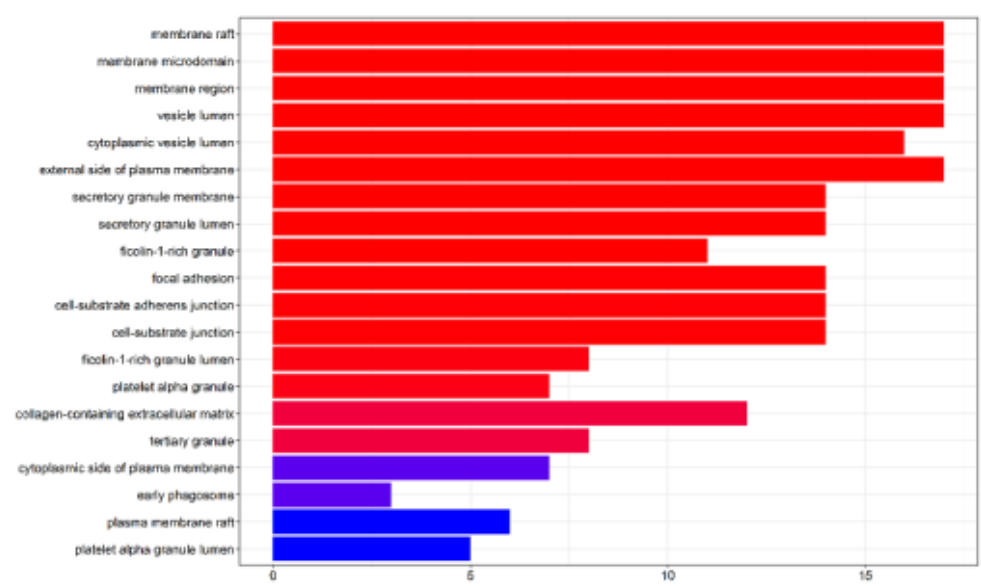

Molecular function

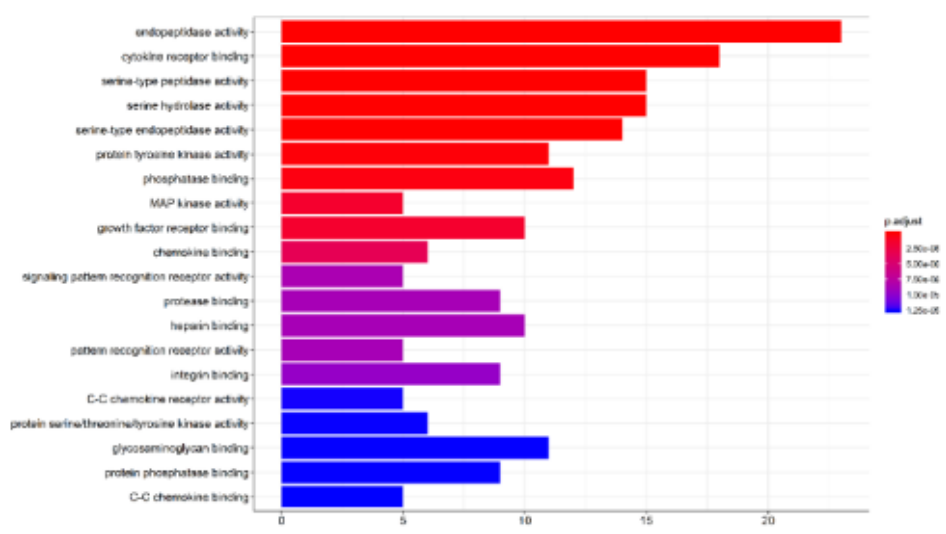

\section{Figure 5}

GO functional analysis of the target proteins, including BP, CC, and MF terms. 
Coronavirus disease - COVID-19 AGE-RAGE signaling pathway in diabetic complications

C-type lectin receptor signaling pathway

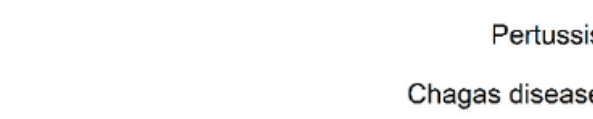

Toll-like receptor signaling pathway

Yersinia infection

Kaposi sarcoma-associated herpesvirus infection-

Tuberculosis

Hepatitis B

TNF signaling pathway

Human cytomegalovirus infection

IL-17 signaling pathway

Toxoplasmosis

Influenza A

T cell receptor signaling pathway

Salmonella infection

PD-L1 expression and PD-1 checkpoint pathway in cancer

Pathogenic Escherichia coli infection-

Measles
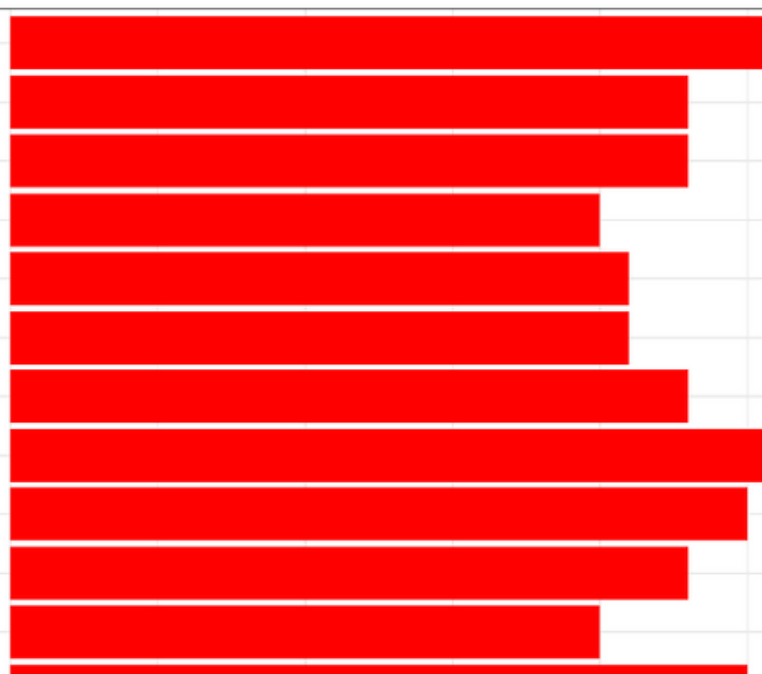

\section{Figure 6}

Results of KEGG pathway enrichment analysis of the overlapped genes. 